\title{
Discoid Medial Meniscus Tear, with a Literature Review of Treatments
}

\author{
In Soo Song, MD, Jun Bum Kim, MD, Jong Keun Lee, MD, and Byeong-Seop Park, MD \\ Department of Orthopaedic Surgery, Daejeon Sun Hospital, Daejeon, Korea
}

The present study reports our experience of treating four cases of symptomatic discoid medial meniscus, three of which were bilateral. We performed partial meniscectomy with a four-portal technique using a knife leaving a $6 \mathrm{~mm}$ peripheral margin after confirmation of magnetic resonance imaging findings. Clinical results were assessed at the end of 2-year follow-up using the Knee Injury and Osteoarthritis Outcome Score and a visual analogue scale. We obtained satisfactory clinical results without recurrence of the symptoms in all cases.

Keywords: Knee, Discoid medial meniscus, Meniscectomy

Young $^{1)}$ reported the first case of discoid lateral meniscus in 1889. Cave and Staples ${ }^{2)}$ described the first two cases of partially discoid medial meniscus in 1941. Murdoch ${ }^{3)}$ reported the first case of bilateral medial discoid menisci in 1956. Since then, more than 23 cases of bilateral discoid medial menisci have been documented to date. The treatment of choice for symptomatic torn discoid meniscus is arthroscopic partial meniscectomy.

The present study reports our experience of treating four cases of symptomatic discoid medial meniscus, three of which were bilateral. The treatment outcomes were assessed with the Knee Injury and Osteoarthritis Outcome Score (KOOS) and a visual analogue scale (VAS).

Received September 2, 2015; Revised (1st) February 18, 2016; (2nd) April 10, 2016; (3rd) May 7, 2016; Accepted May 10, 2016 Correspondence to: In Soo Song, MD

Department of Orthopaedic Surgery, Daejeon Sun Hospital, 29

Mokjung-ro, Jung-gu, Daejeon 34811, Korea

Tel: +82-42-220-8460, Fax: +82-42-220-8460

E-mail: mydangjang@naver.com

This is an Open Access article distributed under the terms of the Creative Commons Attribution Non-Commercial License (http://creativecommons.org/licenses/by-nc/4.0/) which permits unrestricted non-commercial use, distribution, and reproduction in any medium, provided the original work is properly cited.

\section{Case Reports}

\section{Case 1}

A 17-year-old male patient complained of pain in the right knee with locking, giving way and swelling. He did not remember any specific trauma. The symptom duration was 2 years for the right knee and the left knee was asymptomatic. Physical examination of the right knee showed atrophy of the quadriceps femoris muscle compared to the opposite side, medial joint line tenderness and a positive McMurray sign. Plain X-ray showed widening of the medial joint space and cupping of the medial tibial plateau. Magnetic resonance imaging (MRI) of both knees showed complete discoid medial meniscus tears and the right knee demonstrated a horizontal cleavage and flap tear (Fig. 1A and B). We performed partial meniscectomy with a four-portal technique using a knife taking care to preserve the $6 \mathrm{~mm}$ peripheral margin after confirmation by MRI (Fig. 2). The anterior horn of the meniscus was attached to the normal footprint (Fig. 3). At 2 years after surgery, clinical results were assessed by the KOOS and VAS. Any postoperative complications were not seen and the preoperative locking as well as giving way disappeared. The preoperative and postoperative average KOOS were 65.2 and 92.4, respectively. The preoperative and postoperative VAS were 6 and 0 , respectively. 

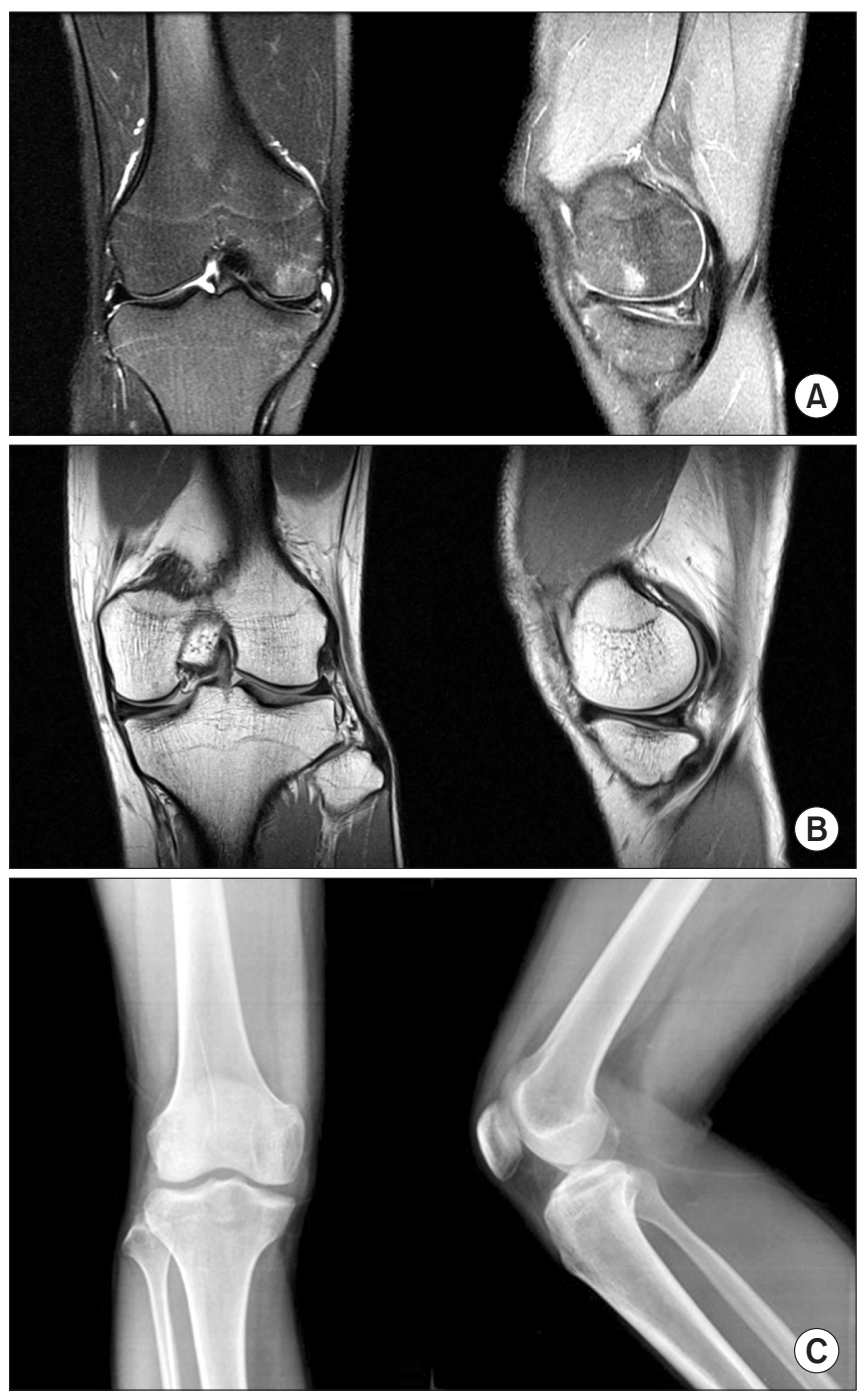

Fig. 1. (A) The right knee magnetic resonance imaging (MRI) showed a complete discoid medial meniscus with continuity of the meniscus from anterior to posterior horns and a horizontal cleavage and flap tear. (B) The left knee MRI showed a complete discoid meniscus, which was treated with conservative management. (C) The plane X-ray showed widening of the medial joint space and cupping of the medial tibial plateau.

\section{Case 2}

A 41-year-old male patient complained of right knee pain. MRI showed a complete discoid medial meniscus with a horizontal cleavage and flap tear in the middle third of the meniscus (Fig. 4). The opposite knee showed a discoid medial meniscus with a tear in the middle portion, but the patient had no complaints on his left knee and physical examination did not reveal any abnormalities (Fig. 5). We tried to preserve the sound meniscus as much as possible to minimal $6 \mathrm{~mm}$ (Fig. 6). The preoperative symptoms disappeared and any complications were not seen. The preopera- tive and postoperative mean KOOS were 75.2 and 91.6, respectively, and the preoperative and postoperative VAS were 5 and 0 , respectively.

\section{Case 3}

A 17-year-old female patient had a slip down injury 3 months before presentation to the clinic. She suffered from continuous swelling and effusion on her right knee with intermittent pain. The range of motion was limited due to the pain during flexion more than $90^{\circ}$. MRI of her right knee showed a complete discoid medial meniscus with a horizontal cleavage and flap tear. We performed a partial meniscectomy. There were no postoperative complications and the preoperative symptoms disappeared. The preoperative and postoperative KOOS were 72.2 and 92.0, respectively. The preoperative and postoperative VAS were 5 and 0 , respectively.

\section{Case 4}

A 40-year-old male patient complained of pain in his right knee with giving way, locking and swelling. The symptom occurred 2 weeks before his visit to the hospital. Physical examination showed medial joint line tenderness and a positive McMurray sign. X-ray showed widening of the medial joint space and cupping of the medial tibial plateau. MRI of the right knee showed a complete discoid medial meniscus with a horizontal flap tear. $\mathrm{He}$ had been diagnosed with a discoid medial meniscus with a tear in the left knee and treated with arthroscopic partial meniscectomy 2 years ago. We performed a partial meniscectomy on the right knee (Fig. 7). There were no postoperative complications and preoperative locking disappeared. The preoperative and postoperative KOOS were 53 and 90.5, respectively, and the preoperative and postoperative VAS were 6 and 0 , respectively.

\section{Discussion}

The discoid meniscus of the knee is an abnormally wide and thick meniscus that widely covers the articular surface of the tibial plateau, and is easily damaged as a result. Discoid lateral meniscus is a well-studied and documented entity of the knee whereas discoid medial meniscus is an extremely rare pathology. There are less than 70 cases of discoid medial menisci reported in the literature ${ }^{4-6}$. The involvement of the bilateral medial menisci is also extremely rare. Dickason et al. ${ }^{6}$ examined 14,731 menisci in a retrospective study and found that of the 8,040 medial menisci (excluding the 6,691 lateral menisci), only 10 were discoid menisci $(0.12 \%)$, and only one had bilateral involvement 

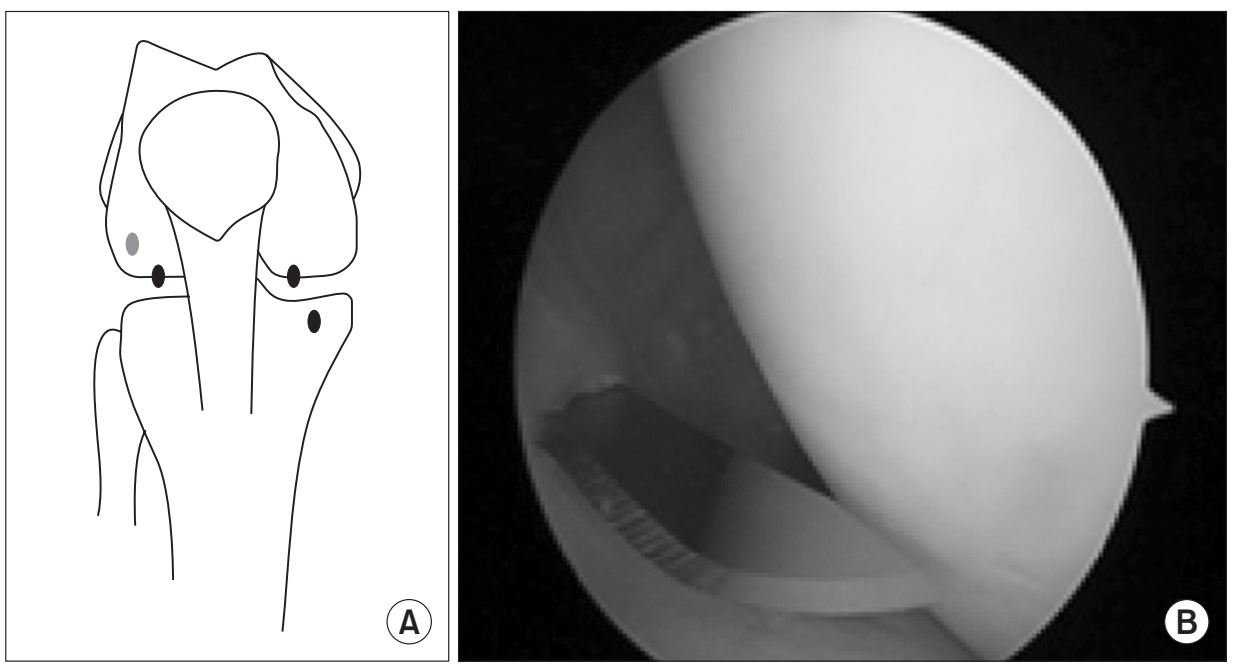

Fig. 2. (A) We used a four-portal technique using the standard anteromedial, anterolateral, additional high far lateral, and additional low anteromedial portals. (B) A No. 15 blade facing anteriorly was inserted into the high far lateral portal and was used to cut the anterior horn of the discoid medial meniscus.
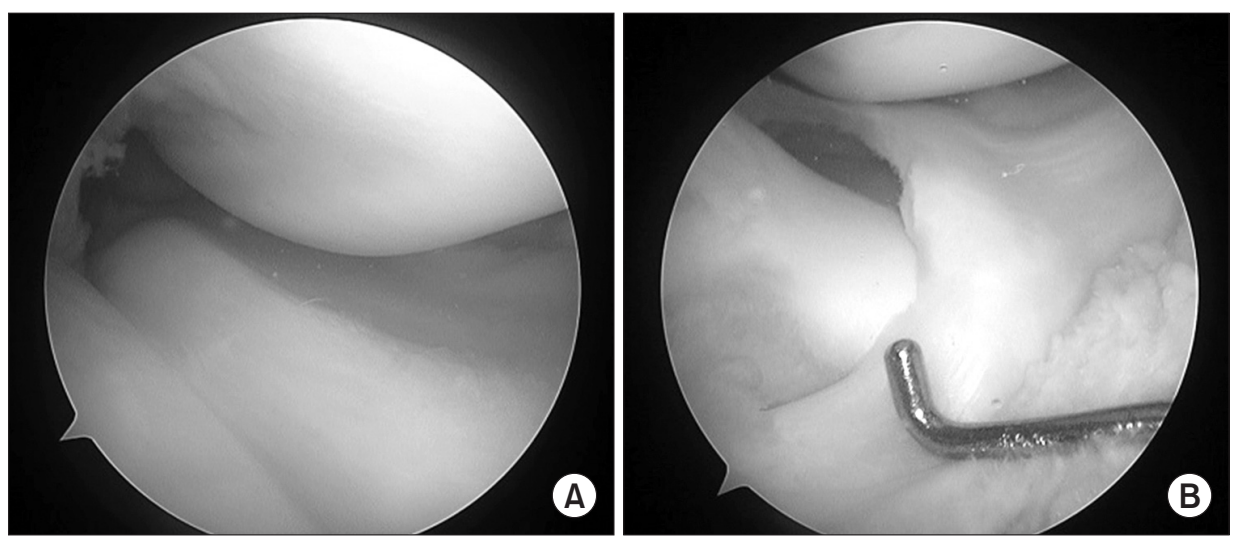

Fig. 3. (A) Arthroscopic findings of a thick complete discoid medial meniscus in a patient. (B) The patient underwent partial meniscectomy with basket punch leaving the $6 \mathrm{~mm}$ stable rim.

$(0.012 \%)$. Although several studies on bilateral discoid medial menisci have been reported by Asian doctors in recent years, no more than 23 cases of bilateral discoid medial menisci have been reported to date. Tachibana et al. $^{4)}$ recommended systematic MRI of the asymptomatic contralateral knee. They considered the incidence of bilateral cases to be underestimated as the abnormality is congenital. Discoid lateral meniscus tends to manifest as hypermobility (snapping knee) in childhood without tear whereas discoid medial meniscus is asymptomatic in childhood until revealed by tear. The ultrastructure of discoid menisci differs from that of normal menisci. These differences in collagen fiber organization and stress redistribution induced by increased meniscal thickness are factors of vulnerability.

Surgical indications are the same for the discoid lateral and medial menisci. Only symptomatic lesions should be operated; in case of preoperative discovery of asymptomatic discoid menisci, abstention is regarded as a rule ${ }^{4)}$. Total meniscectomy had been widely indicated for symptomatic discoid meniscus in the past. Several studies showed excellent results after total menis- cectomy. In 1995, Washington et al. ${ }^{7)}$ reported on the mean 19.8year follow-up results with an average duration of 17 years after total meniscectomy for 18 discoid menisci. They concluded that total meniscectomy might offer the best prognosis with no evident degenerative changes on roentgenograms. Conversely, in 1998, Raber et al. ${ }^{8)}$ retrospectively reviewed long-term results of total meniscectomy for discoid lateral meniscus and reported osteoarthritic changes compared with the untreated contralateral knee in 10 of 11 knees. Therefore, although total meniscectomy was recommended as a treatment for symptomatic discoid lateral meniscus, there was a risk of progressive osteoarthritis and poor prognosis ${ }^{8-10)}$. Therefore, partial meniscectomy (so called "saucerization") is currently recommend for the treatment of symptomatic discoid meniscus to reduce progressive degeneration of the cartilage in the joint. The goal of partial meniscectomy is to remove the central portion of the discoid meniscus and to leave a stable as well as balanced rim. By doing so, the remaining portion performs the function of the meniscus and reduces instability resulting from total meniscectomy. The width of the rim 

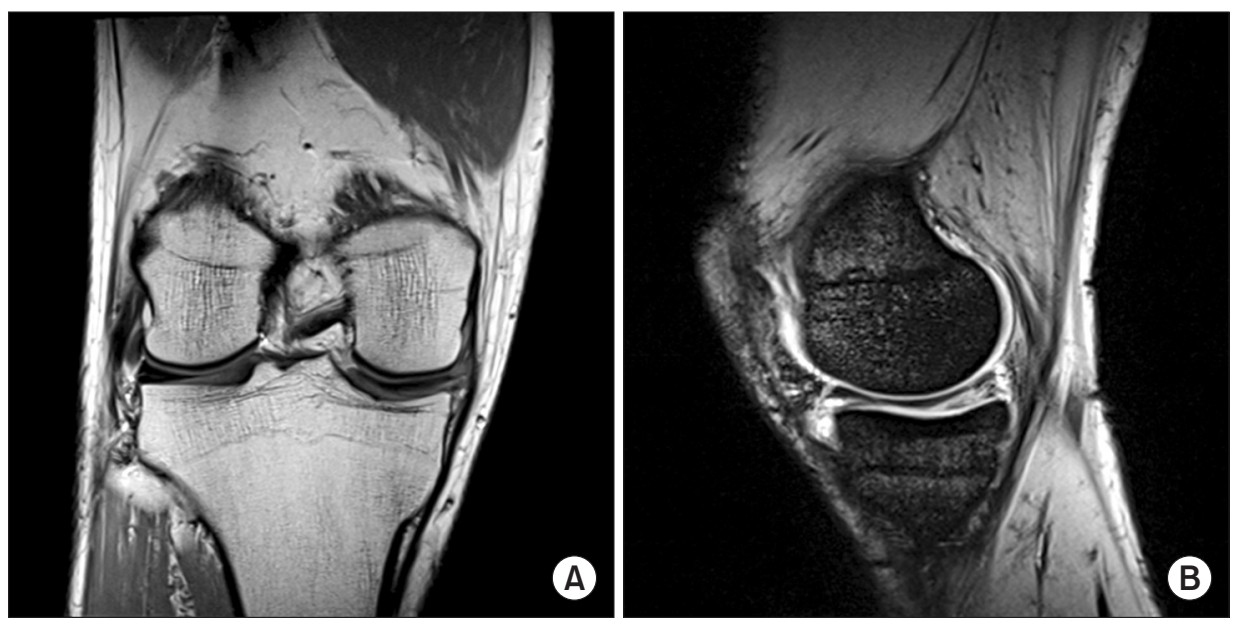

Fig. 4. Right knee magnetic resonance imaging showing a complete discoid medial meniscus of bow-tie configuration with a horizontal cleavage and flap tear.
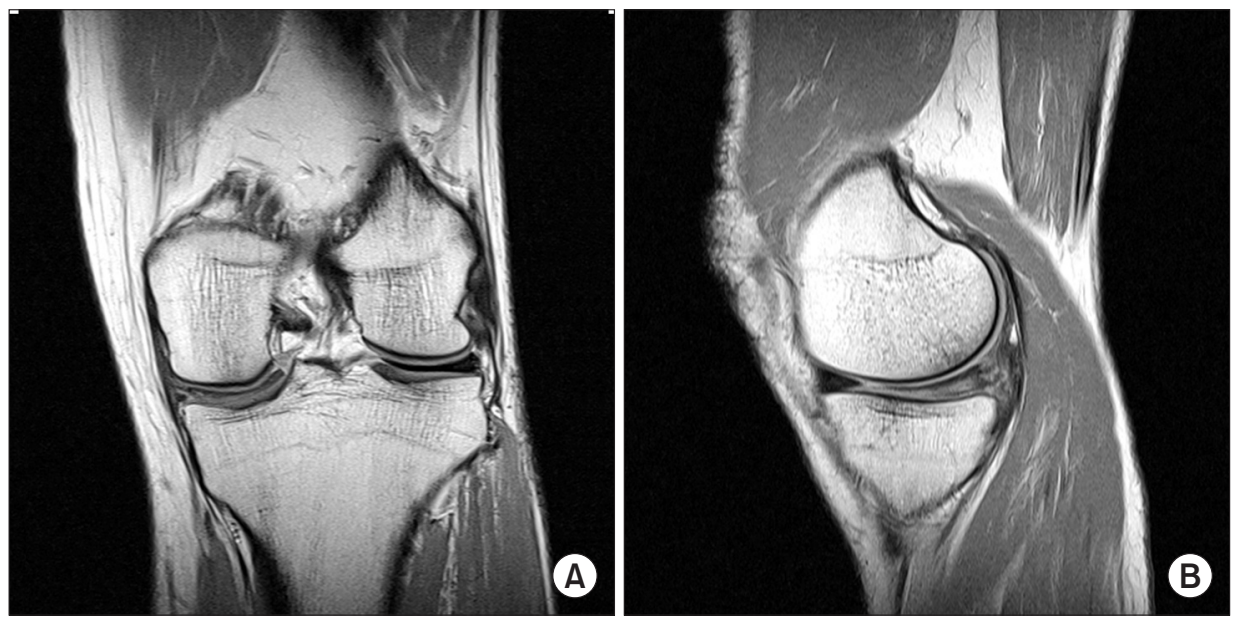

Fig. 5. Left knee magnetic resonance imaging showing a complete discoid meniscus with a horizontal and flap tear in the middle portion.
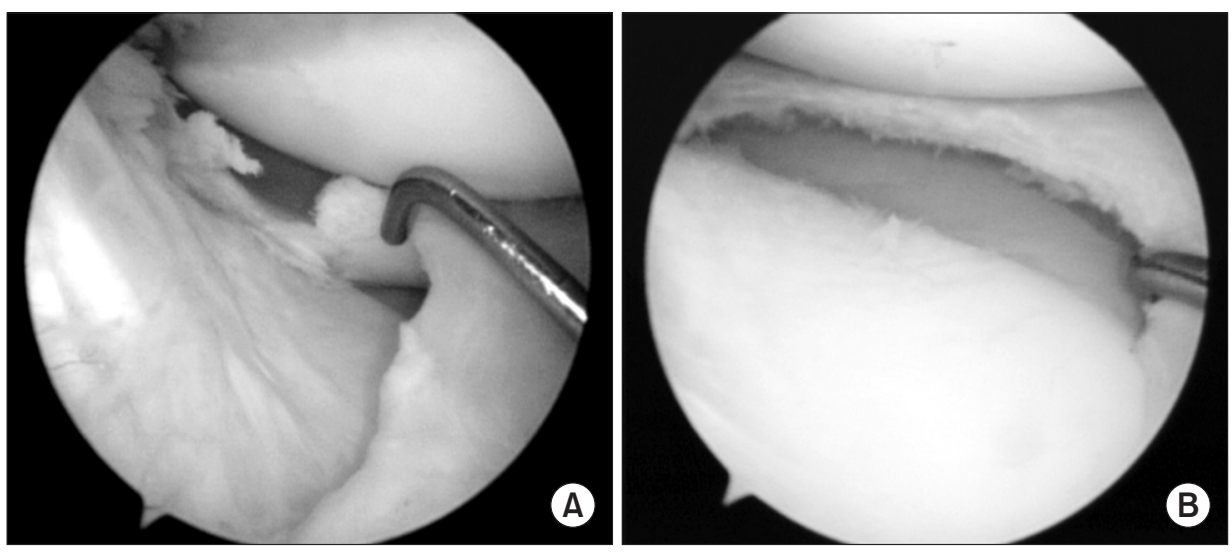

Fig. 6. A complete medial discoid meniscus that underwent reshaping and partial meniscectomy of the inferior flap.

of the remaining meniscus is dependent on the degree of the torn meniscus. The posterior segment and inside the middle segment are known as the most common tear sites of a discoid meniscus. When these sites were torn, Hayashi et al. ${ }^{9)}$ left 6-8 mm width for complete and incomplete lesions and Vandermeer and Cunning- ham ${ }^{10)}$ left 4-5 mm width. In our patients, we used a four-portal technique including the standard anteromedial, anterolateral, additional high far lateral and additional low anteromedial portals. A No. 15 blade facing anteriorly was inserted into the high far lateral portal and was used to cut the anterior horn of the discoidal 

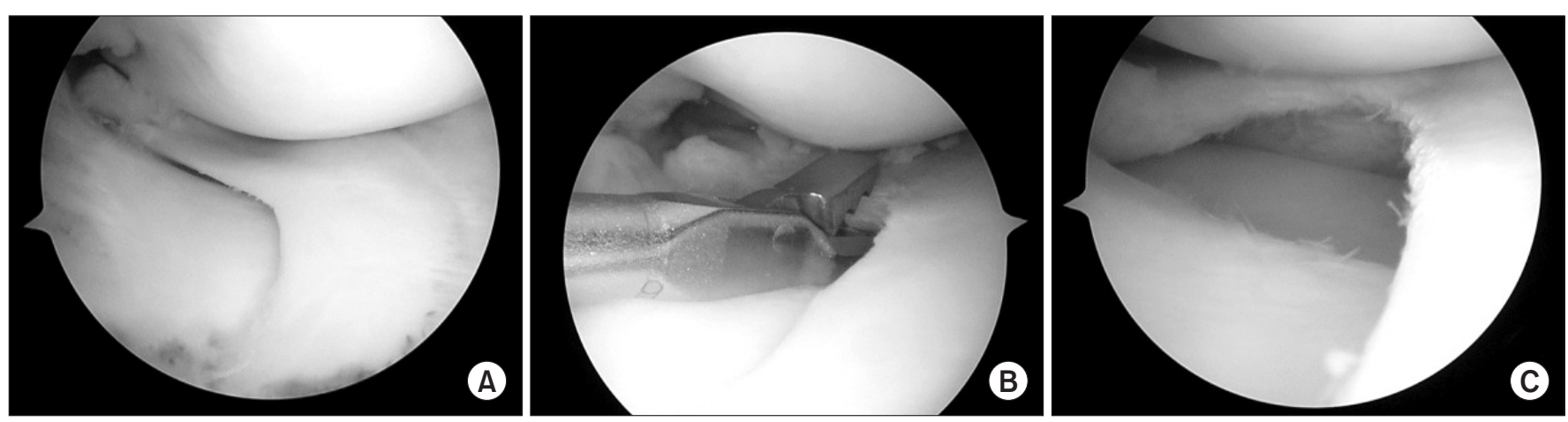

Fig. 7. Arthroscopically confirmed discoid medial meniscus (A) that underwent partial meniscectomy (B) preserving a $6 \mathrm{~mm}$ remnant margin (C).

medial meniscus. Instead of scissors, we used a knife for en bloc resection. As a result, we could determine the remnant margin easily and precisely. Also, we could perform saucerization fast and conveniently without anterior cartilage damage. With this method, we performed partial meniscectomy only on the symptomatic knee, not on the asymptomatic opposite knee and tried to leave a $6 \mathrm{~mm}$ rim to achieve stability and avoid impingement of the femoral condyle against the rim of the meniscus.

Clinically, the most frequent symptoms of discoid medial meniscus are medial knee pain, iterative effusion and locking in flexion which are not specific ${ }^{4-6}$. Knee snapping is relatively rarer in discoid medial meniscus than in discoid lateral meniscus. Pain and effusion are more associated with the meniscal tear rather than the discoid shape of the meniscus. Locking may occur because of the discoid shape as such, with the thick central region passing forward of the medial condyle.

Plain X-ray may show an enlarged joint space in the knee with a discoid medial meniscus ${ }^{5}$. MRI is the best tool for confirming diagnosis and exploring the associated meniscal lesions. Diagnosis of discoid medial meniscus requires continuity between the anterior and posterior horns on three consecutive 5-mm thick sagittal slices and is confirmed by coronal slices showing an abnormally thick meniscus, sometimes extending as far as to the intercondylar groove ${ }^{4}$. Our patients had radiological evidence of discoid medial meniscus described in the literature, such as hypoplasia of the medial tibia and femoral condyles and increased medial joint space. But none of them had a lytic lesion in the distal femoral condyle. Both MRI and arthroscopy did show the presence of a depressed medial tibia plateau and complete discoid medial meniscus with continuity from the anterior horn to the posterior horn.

We think that discoid medial meniscus tears should be managed with more active surgical treatment because the medial meniscus is subject to greater stress due to screw home mechanism and less mobility. Furthermore, because weight bearing is transferred to the medial side of the knee, patients tend to have more discomfort, such as giving way, locking and pain than patients who have lateral discoid meniscus tears.

In conclusion, we experienced four cases of symptomatic discoid medial meniscus and managed the rare deformity using a four-portal arthroscopic partial meniscectomy, which is similar to the technique used in knees with a discoid lateral meniscus. We think that active surgical treatment for a torn discoid medial meniscus can provide satisfactory results without recurrence of symptoms.

\section{Conflict of Interest}

No potential conflict of interest relevant to this article was reported.

\section{References}

1. Young RB. The external semi-lunar cartilage as a complete disc. In: Cleland J, MacKay JY, Young RB, eds. Memoirs and memoranda in anatomy, vol. 1. London: Williams and Norgate; 1889. p179.

2. Cave EF, Staples OS. Congenital discoid meniscus: a cause of internal impingement of the knee. Am J Surg. 1941;54:371-6.

3. Murdoch G. Congenital discoid medial semilunar cartilage. J Bone Joint Surg Br. 1956;38:564-6.

4. Tachibana Y, Yamazaki Y, Ninomiya S. Discoid medial meniscus. Arthroscopy. 2003;19:E12-8.

5. Pinar H, Akseki D, Karaoglan O, Ozkan M, Uluç E. Bilateral discoid medial menisci. Arthroscopy. 2000;16:96-101.

6. Dickason JM, Del Pizzo W, Blazina ME, Fox JM, Friedman MJ, Snyder SJ. A series of ten discoid medial menisci. Clin Orthop Relat Res. 1982;(168):75-9. 
7. Washington ER 3rd, Root L, Liener UC. Discoid lateral meniscus in children: long-term follow-up after excision. J Bone Joint Surg Am. 1995;77:1357-61.

8. Raber DA, Friederich NF, Hefti F. Discoid lateral meniscus in children: long-term follow-up after total meniscectomy. J Bone Joint Surg Am. 1998;80:1579-86.
9. Hayashi LK, Yamaga H, Ida K, Miura T. Arthroscopic meniscectomy for discoid lateral meniscus in children. J Bone Joint Surg Am. 1988;70:1495-500.

10. Vandermeer RD, Cunningham FK. Arthroscopic treatment of the discoid lateral meniscus: results of long-term followup. Arthroscopy. 1989;5:101-9. 